\title{
Point de Vue Interne du Diable au Corps de Raymond Radiguet
}

\author{
Raymond Radiguet'nin İçimizdeki Şeytan'ının İç Bakış Açısı
}

\section{Songül ASLAN KARAKUL*}

Résumé: L'objectif de cet article est de mettre en évidence la notion du point de vue (ou la focalisation) interne dans le Diable au Corps de Raymond Radiguet ${ }^{1}$ Il est essentiel, au lecteur, de différencier l'auteur du narrateur car ces deux termes sont la plupart du temps confondus. Ce serait le narrateur, pas l'auteur, à prendre en considération. Dans ce texte narratif, le narrateur raconte l'histoire à l'aide d'une focalisation interne choisie selon laquelle la perception du récit peut en dépendre. "Le sens est fondé sur la différence: il y a du sens lorsqu'il y a de la différence" (Groupes d'entrevernes 1979, 13). A la focalisation interne, le narrateur est le narrateur de l'histoire, dont il est lui-même le protagoniste ou le témoin. On limiterait à une étude approfondie de la focalisation interne primordiale dans le récit intitulé le Diable au corps. Dans ce type de focalisation, le narrateur du récit est celui qui est de premier degrés racontant (au lecteur virtuel) une histoire dans laquelle il est présent comme personnage (protagoniste ou témoin). Ceci sera présenté tout au long du travail à travers des arguments et des exemples empruntés dans le récit.

Mots-clés: Point de vue, Focalisation, Focalisation Interne, Diable au Corps, Auteur/Narrateur, Récit

Öz: Bu makalenin amacı, Raymond Radiguet'nin Le Diable au Corps adlı eserinin bakış açısı (iç odaklayım) kavramını ele almaktır. Burada öncelikle anlatıcı kavramını yazar kavramından ayırdetmek gerekir. Zira, okuyucu bu iki kavramı çoğunlukla birbiriyle karıştırmaktadır. Anlatı incelemesinde dikkate alınacak kişi yazar değil anlatıcı olacaktır. Bu metinde, anlatııı hikayeyi iç odaklayım ile anlatmaktadır. Bu da şeçilen bu odaklayıma göre hikayenin algılanmasını sağlar. "Anlam farklllkklar üzerine kuruludur: Ĕger farklllık varsa anlam vardır" (Groupes d'entrevernes 1979, 13). İç odaklayımda, anlatıcı kendisinin de içinde başkahraman ya da tanık olarak bulunduğu hikayenin, birinci dereceden anlatıcısıdır. Çalışma le Diable au Corps' un iç odaklayımını kapsamlı bir biçimde ele almayla sınırlandırılmıştır. Bütün çalışma boyunca metinden örneklerle birinci dereceden anlatıcı özelliği olan iç odaklayım kanıtlanmaya çalışlacaktır.

Anahtar sözcükler: Bakış Açısı, Odaklayım, İç Odaklayım, Diable au Corps, Yazar/Anlatıcı, Anlatı

\section{Introduction}

La composition d'un roman est très importante, car elle conditionne la lecture, elle contribue à conduire le lecteur là où l'auteur le veut. Non seulement elle donne cohérence et efficacité au roman, mais elle a aussi une fonction esthétique et d'équilibre, à l'intérieur du roman. Avant d'élargir nos propos, ce serait mieux de différencier le narrateur de l'auteur. Jean-Pierre Goldenstein dirait ainsi qu'il faut premièrement réfléchir sur ce qui se passe dans un récit tout en répondant à cette fameuse question "qui parle?" (28). "L'auteur est celui qui imagine l'oeuvre

\footnotetext{
* Maître de conférence adjoint, Université Adnan Menderes, Faculté des Siences et des Lettres, Département de la Langue et Littérature françaises. songulaslankarakul@gmail.com
} 
(roman, nouvelle, etc...), le narrateur est celui qui raconte l'histoire. “... qui parle (dans le récit ) n'est pas qui écrit (dans la vie) et qui écrit n'est pas qui est" (Goldenstein rapportant de Barthes 1980, 28). L'auteur est le concepteur, le narrateur est la voix choisie pour raconter l'histoire. Dans les romans à la première personne, le "je" correspond ainsi au narrateur et pas à l'auteur. Afin de démontrer cette réalité, il suffit de lire un extrait expliquant un suicide ou la mort tout simplement. Comment une personne ayant rendu son âme peut-elle nous décrire son ressenti, sa vision lorsqu'elle était en train de mourrir si elle n'a pas survécu. Il est donc important de réaliser que "l'auteur est la personne réelle qui vit ou a vécu en un temps et en des lieux donnés; a pensé telle ou telle chose; peut faire l'objet d'une enquête biographique, inscrit généralement son nom sur la couverture du livre que nous lisons" (Goldenstein 1980, 29). Quant au narrateur c'est celui qui raconte la fiction choisie par l'auteur. Il s'agit donc d'une "voix de papier" ("La narration peut être caractérisée par la dissociation du narrateur et de la personne biographique de l'auteur. Deux instances médiatrices caractérisent le discours romanesque: le narrateur (acteur-narrateur jouant un rôle dans l'histoire racontée ou auteur-narrateur anonyme ne participant pas à l'action) et l'auteur abstrait (déduit du texte, à la différence de l'auteur concret)" Adam J.-M. 1990, 173).

Pour préciser ce que nous argumentons à propos de cette différence, sur le schéma de l'énonciation ci-dessous est empruntée partiellement de Guy Spielmann (Speilmann, consulté: le 3 Février 2018) :

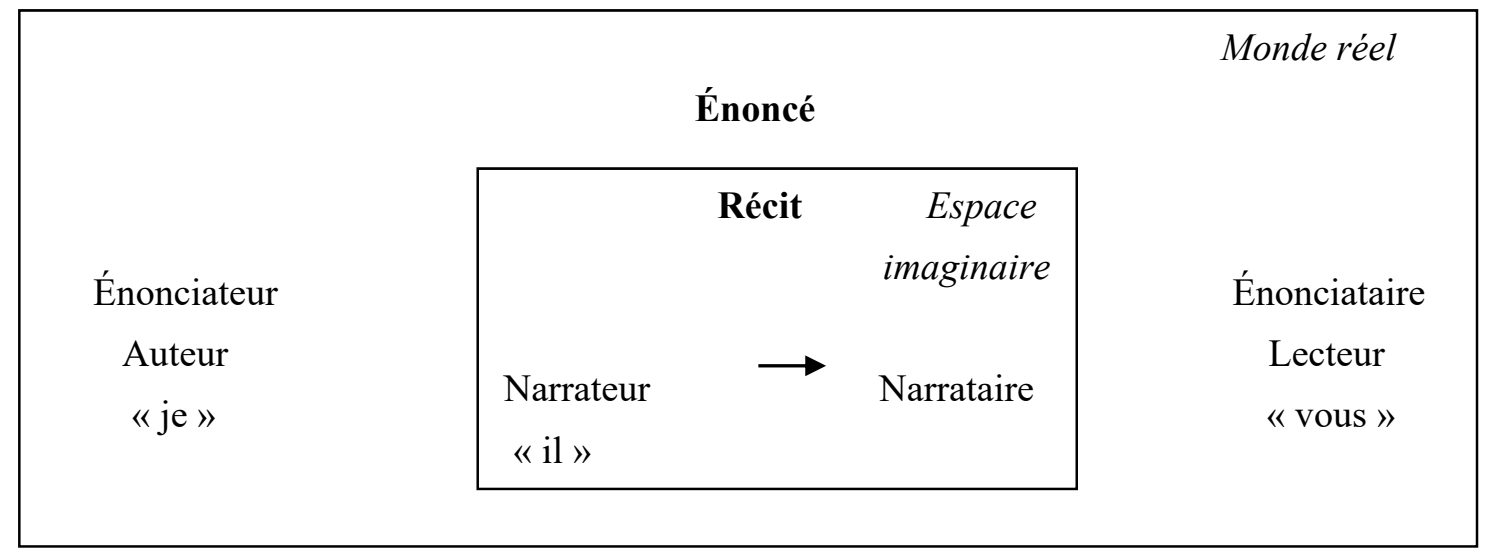

Dans ce schéma, le monde réel représente notre univers regroupant tous les vivants ou nonvivants. Tandis que l'espace imaginaire n'appartient qu'aux personnages du récit. Celui-ci, est l'équivalent de l'énoncé (coupé de la situation de l'énonciation). Dans ce cas, l'énonciateur dans le monde réel sera celui qui imagine l'histoire, c'est-à-dire l'auteur. Il chargera en donnant la parole à un de ces personnages dans son espace imaginaire et prendra du recul afin de permettre au narrateur de diriger l'histoire du récit. Le narrateur assumera désormais tous les actes. La responsabilité n'appartient donc plus à l'auteur. Tout en tenant compte que l'énonciateur transmet son énoncé à un énonciateur. C'est un cas semblable dans l'espace imaginaire, le narrateur raconte son histoire à un narrataire virtuel bien entendu.

Notre première tâche ainsi accomplie à l'aide du tableau ci-dessus, nous pouvons nous libérer de l'auteur afin d'analyser le point de vue du narrateur dans notre récit en question. En effet, c'est sur lui que nous nous focaliserons. On peut enchaîner avec une vue d'ensemble à ce sujet.

\section{Focalisation}

Les romans sont toujours, malgré leur variété, des récits d'événements situés qui font intervenir 
des personnages et des rapports de succession logique et chronologique. Le narrateur introduit ainsi dans la communication une histoire qui peut prendre son autonomie, derrière laquelle il peut s'effacer, mais qu'il lui est loisible, à tous moments de commenter. Le genre romanesque joue donc naturellement, plus que les deux précédents, sur la superposition des axes énonciatifs. Par le choix des focalisations (le terme "focalisation est employé pour déterminer le lieu d'où le narrateur relate le récit ou les descriptions des faits dans une oeuvre romanesque. La focalisation révèle les différents foyers qui permettent de décrire les événements, les paysages ou les personnages. Donc, il s'agit de désigner celui qui voit ou celui qui perçoit dans la focalisation (Özel Coulibaly \& Işın 2012, 60), des voix narratives et l'introduction des paroles de personnages, le narrateur peut, en effet, établir avec le lecteur une communication plus ou moins directe. "On peut en effet raconter plus ou moins ce que l'on raconte, et le raconter selon tel ou tel point de vue; et c'est précisément cette capacité et les modalités de son exercice, que vise notre catégorie du mode narratif" (Genette 1972, 183).

Le choix du mode narratif du récit peut transmettre au lecteur des détails directes ou non et semble de cette façon mettre une distance à ce qu'il raconte. Le récit "peut aussi choisir de régler l'information qu'il livre, non plus par cette sorte de filtrage uniforme, mais selon les capacités de connaissances de telle ou telle partie prenante de l'histoire (personnage ou groupe de personnages)" (Genette 1972, 183-184).

la "représentation" ou l'information narrative

Le terme focalisation sert à désigner, à la suite de G. Genette, la délégation faite par l'énonciateur (auteur) à un sujet cognitif, appelé observateur (narrateur), et son installation dans le discours narratif: cette procédure permet ainsi d'apréhender soit l'ensemble du récit, soit certains programmes pragmatiques seulement du "point de vue" de ce médiateur (Greimas et Courtès 1993, 150).

Les types de cette installation sont ainsi indiqués sur le tableau suivant qui précise le cas en guise de renseignement supplémentaire (Limoges 2013).

\begin{tabular}{|l|l|l|}
\hline & \multicolumn{1}{|c|}{ Extra diégétique } & \multicolumn{1}{|c|}{ Intra diégétique } \\
\hline Hétérodiégétique & $\begin{array}{l}\text { Narrateur de premier degrés racontant } \\
\text { (au lecteur virtuel) une histoire de } \\
\text { laquelle il est absent comme } \\
\text { personnage. }\end{array}$ & $\begin{array}{l}\text { Narrateur de second degrés racontant (à } \\
\text { d'autres personnages de l'histoire) une } \\
\text { histoire de laquelle il est absent. }\end{array}$ \\
\hline Homodiégétique & $\begin{array}{l}\text { Narrateur de premier degrés racontant } \\
\text { (au lecteur virtuel) une histoire dans } \\
\text { laquelle il est présent comme } \\
\text { personnage (protagoniste ou témoin). }\end{array}$ & $\begin{array}{l}\text { Narrateur de second degrés racontant (à } \\
\text { d'autres personnages de l'histoire) une } \\
\text { histoire dans laquelle il est présent } \\
\text { comme personnage (protagoniste ou } \\
\text { témoin). }\end{array}$ \\
\hline
\end{tabular}

- "Le narrateur personnage (ou intérieur au récit ou homodiégétique) : le récit est à la première personne et le narrateur est un des personnages, personnage principal et secondaire qui prend en charge le récit.

- Le narrateur extérieur au récit (ou hétérodiégétique) : il correspond à une voix indéterminée qui n'est pas celle d'un personnage, le récit est à la 3ème personne.

Même extérieur au récit, le narrateur peut intervenir dans la narration : explicitement par des interventions (jugements sur les personnages, sur 
l'action; apostrophes au lecteur ...), implicitement par le choix d'un vocabulaire mélioratif ou dépréciatif , par l'ironie..." (Jocelyne, consulté le 05 Février 2018).

On distingue donc dans un roman trois types de focalisations mais il n'est pas rare qu'il y ait succèssivement plusieurs point de vue dans un même texte.

\section{Focalisation zéro (vision illimitée)}

La focalisation zéro est très souvent dans les romans classiques ceux de J. Verne ou de Balzac par exemple, le lecteur a l'impression que le narrateur sait tout et voit tout. Il peut suivre les personnages partout à la fois et connaît leurs pensées les plus secrètes. En d'autres termes, "le narrateur délivre au lecteur des informations dont ne disposent pas les personnages. Il a une vision d'ensemble et peut décrire des évenements qui se déroulent simultanément dans des endroits différents. Il peut informer sur l'avenir du personnage" (Jocelyne, consulté le 5 Février 2018).

Son champ de vision est alors infini; on parle à ce propos de focalisation zéro, c'est-à- dire qu'il n'y a pas de point de vue privilégié, pas de foyer optique précis. L'habitude des conventions romanesques fait que le lecteur ne s'étonne pas de cette omniscience pourtant peut vraisemblable du narrateur, et lit sans surprise des passages comme celui- ci: au début de L'Île mystérieuse, les naufragés ont été jetés par la tempête sur une île, mais il manque l'un des passagers du ballon, l'ingénieur Cyrus Smith. Le narrateur écrit alors:

Cependant le ciel se dégageait peu à peu. Vers minuit, quelques étoiles brillèrent, et si l'ingénieur eût été là, près de ses compagnons, il aurait pu remarquer que ces étoiles n'étaient plus celles de l'hémisphère boréal (Verne, Consulté, le 4 Février 2018, 22).

L'avantage de cette vision est le fait que le narrateur a la capacité de se trouver à plusieurs endroits simultanément tout en connaissant les pensées les plus secrètes des personnes. Même si le narrateur se promène dans tous les coins du récit en même temps, dans les sentiments de tous les personnages de la fiction le lecteur n'est pas étonné car à la lecture de la première ligne il est prêt être dupé à travers du récit.

\section{Focalisation externe}

Il s'agit de la focalisation externe lorsque le point de vue est celui d'un observateur extérieur et objectif, qui ne fait que constater ce qui est visible du dehors, sans explications ni commentaires:

En 1829, par une jolie matinée de printemps, un homme âgé d'environ cinquante ans suivait à cheval un chemin montagneux qui mène à un bourg situé près de la Grande-Chartreuse [...]. Homme de haute taille, le voyageur était entièrement vêtu de drap bleu aussi soigneusement brossé que devait l'être chaque matin son cheval au poil lisse, sur lequel il se tenait droit et vissé comme un vieil officier de cavalerie (Balzac 1874, 20).

"Le narrateur ne décrit ou raconte que comme le ferait un observateur : le lecteur n'accède ni aux pensées, ni aux sensations, ni aux sentiments des personnages. Il ne les connaît que par leurs gestes, leurs paroles et leurs actes" (Jocelyne, consulté le 03 Février 2018).

Bien sûr, comme nous l'avons déjà cité, il peut y avoir des variations dans le champ de vision à l'intérieur d'un même récit. Par exemple, Balzac passe ensuite à la focalisation zéro 
pour expliquer au lecteur qui est le personnage en question. Dans le roman de Maupassant, le champ de vision, après avoir coïncidé pendant tout le début du récit avec le personnage de Pierre, se déplace ensuite de façon significative vers d'autres personnages.

\section{Focalisation interne (vision limitée)}

Dans la focalisation interne, le champ de vision peut être plus restreint si le narrateur s'identifie à un personnage et ne raconte que ce que celui-ci sait, voit, sent. On parlera alors de focalisation interne pour ce personnage, dont le point de vue se trouve privilégié:

"Pierre se dirige vers la rue de Paris, la principale rue du Havre, éclairée, animée, bruyante. L'air un peu frais de bords de mer lui caressait la figure, et il marchait lentement, la canne sous le bras, les mains derrière le dos. Il se sentait mal à l'aise, alourdi, mécontent comme lorsqu'on a reçu quelque fâcheuse nouvelle..."

Dans ce cas, le narrateur ne décrit ou raconte que ce que voit, ressent, pense ou connait un personnage (ou plusieurs personnages succéssifs). Le lecteur perçoit la scène vue, ressentie par ce personnage (ou ces personnages). Selon Alexandrescu, la division des rôles entre le narrateur et l'auteur en ce qui concerne le Je va avoir un impact sur le plan de ses (auto) qualification (p. 62)

\section{La focalisation interne du Diable au corps}

Le Diable au corps est écrit à la première personne, "je" et le roman se présente comme une confession rétrospective. Le narrateur est le héros de l'histoire, et tout est raconté de son point de vue. Les autres personnages ne nous apparaissent que dans ce qu'il perçoit d'eux. Comme l'indique V. Doğan Günay (2013:476), pour décrire leurs actes et leurs paroles il n'utilise pas le style direct en employant des guillemets mais opte pour le style indirect. Ainsi ne connaissonsnous les sentiments de Marthe que par l'analyse qu'en fait le jeune homme, et les jugements qu'il porte sur ses parents ou les voisins et les relations sont les seuls éléments que nous possédions pour essayer de nous en faire une idée.

Ce choix narratif, la focalisation interne, pose le problème de communication au lecteur d'informations que le narrateur, n'est pas censé connaître, comme les pensées des autres personnages par exemple. Raymond Radiguet fait appel à quatre solutions:

1. Le narrateur apprend plus tard la vérité sur une attitude, un sentiment: “C'était d'ailleurs vrai, simplement, je le sus dans la suite, parce que Marthe avait faussé notre conversation dans le même sens que moi" (34).

2. Le narrateur se fie à une impression qui peut être fausse: “... je gardai pour moi que d'après cette scène il était impossible que Marthe ne fût pas méchante" (83-84).

3. Le narrateur prend des mesures pour formuler des hypothèses: "Le lendemain, comme nous traversions le jardin, il bêchait. Sans doute était-ce un pensum..." (104).

4. Le narrateur donne des explications psychologiques simples: "[Ma mère] était jalouse.

Elle regardait Marthe avec des yeux de rivale. Elle trouvait Marthe antipathique, ne se rendant pas compte que toute femme, du fait de mon amour, le lui serait devenue" (8 8-89).

Cette dernière solution est la plus employée parce qu'elle permet de garder l'unité du récit, en même temps que sa vraisemblance. L'efficacité psychologique du narrateur s'exerce sur des personnes dont il a pu observer le comportement. Raymond Radiguet respecte ainsi la logique et l'unité de la focalisation interne. C'est pourquoi le personnage de Marthe reste une énigme autant pour le lecteur que pour le narrateur:

"[...] et je souffrais de ne pouvoir pénétrer son esprit" (87) 
Comme nous le savons, ce qui est raconté est perçu et interprété d'un point de vue d'un personnage précis. La réalité décrite est limitée par une perception subjective d'un narrateur qu'il soit un personnage ou non.

Raymond Radiguet adopte le point de vue d'un narrateur-écrivant qui rapporte ses actions antérieures et ouvre ainsi la voie à l'analyse psychologique: le narrateur-écrivant $(\mathrm{X})$ cherche à comprendre - et parfois à justifier - les actions du narrateur-personnage (x). Donc, de X à x le personnage a évolué et peut juger de ses erreurs. Des formules récurrentes rappellent les étapes de cet apprentissage:

Il a acquis une expérience qu'il ne possédait pas.

"J'ignorais que servitude pour servitude, il vaut encore mieux être asservi par son cœur que l'esclave de ses sens" (142-143).

Il a mesuré ses erreurs, comme l'atteste la fréquence du verbe "croire" à l'imparfait.

"Nous nous croyions des hommes" (26).

“je croyais avoir des raisons profondes [...]. je croyais qu'il s'était passé des choses graves [...] je croyais avoir déclaré mon amour à une personne insensible" (34).

"je me croyais renvoyé du collège mais je me trompais..." (52) "je croyais que l'amour ne peut se passer." (56) "[...] moi qui me croyais un homme ..." (67) "Je croyais certains mensonges commandés par le code passionnel" (141) "je croyais la lui devoir, par dignité" (182).

Ces exemples, répartis sur tout le roman du début à la fin, montrent que l'apprentissage du narrateur est marqué par des erreurs et que la maturité naît des errements passés. Bien entendu, tirer une conséquence de sa jeunesse semble innocenter le narrateur; pourtant cette accumulation d'erreurs, son aveuglement indiquent l'infatuation, la vanité de la jeunesse (34-35).

D'ailleurs, la démarche du narrateur pour inviter Carmen est plus un plan préparé, avec des complices ménagées plus longtemps qu'une folie d'amoureux. Dans cet amour enfantin, nous relevons déjà un conflit entre les sentiments et la raison: c'est la raison qui l'importe. Dans 1' "aventure" avec Marthe, le conflit sera plus violent et la fin beaucoup plus dramatique. L'Episode de Carmen a un autre aspect du point de vue des rapports que le narrateur entretient avec la morale.

En effet, confronté au monde des adultes et à la société, le narrateur prend conscience que certaines règles sont floues, obéissant davantage à la convenance qu'à la rigueur morale:

"Ce qui troubla fort mes notions de morale, fut qu'il considérait comme aussi grave d'avoir compromis la jeune fille (dont les parents lui avaient communiqué ma déclaration), que d'avoir dérobé une feuille de papier à lettres (10).

D'autre part, son aventure, au lieu d'attirer l'hostilité des camarades de classe, lui donne du prestige:

“[...]. Le professeur, ironique, m'appela Don Juan. J'en fus extrêmement flatté [...]. Son "Bonjour, Don Juan" et mon sourire entendu transformèrent la classe à mon égard" (10).

En effet, le narrateur est très attaché à certaines valeurs et il est très surpris que Marthe ne les partage pas. Par exemple, alors que Marthe n'est pas allée guetter le train dans lequel était Jacques partant pour l'hôpital de Bourges, le narrateur, lui, se serait montré, sincèrement, conciliant et compatissant.

"Je lui expliquai ma réserve, mon respect pour ses devoirs envers Jacques malade. Elle me 
crut à demi. J'étais irrité. Je faillis lui dire: "Pour une fois que je ne mens pas" (118).

Ainsi, leur liaison du point de vue de la morale se trouve justifiée peut-être d'une manière spécieuse. Mais ce genre de raisonnement ne suffit pas à calmer le malaise du narrateur. Entraîné loin par l'amour, il ne demande qu'à rentrer dans le rang.

Le narrateur se laisse emporter par la puissance invincible de l' "ordre". Il éprouve une double satisfaction: la première est une satisfaction d'amour et d'amour-propre; la seconde est une satisfaction morale. Là encore nous retrouvons le conflit entre amour et raison. Mais l'objet de l'amour est la mort et c'est la raison qui triomphe. De plus, la raison n'a pas une place privilégiée, la dernière, étant donné que le roman s'achève par le mot "raisonnable".

Le Diable au corps est composé d'épisodes; et quand nous fermons le livre, nous revoyons Marthe et le narrateur à Paris, sous la pluie, amants pitoyables à la recherche d'un hôtel; nous repensons à la première nuit et à la cruauté du narrateur; nous nous rappelons le raout des Marin, le suicide de la bonne des Maréchaud, l'achat des meubles, la détresse de Jacques, l'annonce de la mort de Marthe... Tous ces épisodes sont organisés entre eux de manière rigoureuse par l'auteur, pour donner au récit cohérence et séduction: on appelle d'habitude cette organisation des épisodes du récit, la "composition dramatique" (4).

Selon la recherche, ne s'intéresser au Diable au corps que pour ces raisons mentionnées cidessus serait injuste. Il est à noter que c'est un roman inépuisable dont les lectures et les relectures successives relèvent sans cesse de certains nouveaux aspects, de nouvelles subtilités peu rencontrés jusqu'alors. C'est aussi un roman intéressant du point de vue de la narration, la composition et la structure. Il va sans dire que c'est un roman initiatique. C'est le récit d'une métamorphose: une aventure bouleverse la vie d'un adolescent et le transforme en adulte. Le narrateur, le personnage principal, évolue et réagit en fonction de ses rencontres, de son entourage, des événements extérieurs. Dans cet univers fictif, personnages et contexte sont inséparablement liés.

\section{Conclusion}

En adoptant le point de vue d'un narrateur-écrivant rapporteur de ses actions antérieures, Raymond Radiguet ouvre la voie, à l'analyse psychologique. Tout cela nous montre que l'apprentissage du narrateur est ponctué d'erreurs, et que la maturité naît des errements passés. En effet, un jeune garçon fait ses premières armes amoureuses avec une jeune femme plus âgée que lui, c'est la situation classique du roman d'apprentissage.

Ce travail a pu mettre le point sur la différence auteur-narrateur grâce au contexte (discours et récit). Suite à cette distinction, cet article s'est porté sur l'indentification de la focalisation du narrateur et l'importance du point de vue à percevoir la bonne signification du texte. On choisi de traiter la focalisation interne en tant qu'un des éléments constitutifs de l'analyse narrative de texte, dans le Diable au corps. Raymond Radiguet respecte ainsi la logique et l'unité de la focalisation interne. C'est pourquoi le personnage de Marthe reste une énigme autant pour le lecteur que pour le narrateur. Dans la focalisation interne, le point de vue limité du narrateurprotagoniste lui a servi d'apprendre et de mûrir en faisant des erreurs. La lecture du récit à travers le regard du narrateur nous permet d'avoir une approche sur l'évolution du personnage qui nous est présentée comme une succession d'états (Groupes d'entrevernes 1979, 13). Par conséquant, pour chaque oeuvre il est essentiel au lecteur d'analyser sous quel angle les faits sont apperçus. En effet, cette vision nous permet de nous demander si elle est unique ou si elle change durant la narration et dans quelle intention. 


\section{Note de l'auteur}

Article produit d'une partie de la thèse de doctorat inédite "Procédés narratifs dans le Diable au corps de Raymond Radiguet: une approche sémio-linguistique" de Songul Aslan Karakul sous la direction de Professeur Zeynel Kıran, présentée en 2008.

\section{BIBLIOGRAPHIE}

Adam J. M. (1990). Le Texte narratif. Paris 1990.

Alexandrescu S. (1973). "Le Discours Etrange: essai de définition à partir d'une anlyse de "La Nuit" de Maupassant". Sémiotique narrative et textuelle. Présenté par Chabrol, C. Paris (1974) 55-95.

Aslan-Karakul S. (2008). Procédés narratifs dans le Diable au corps de Raymond Radiguet: une approche sémio-linguistique. Université Hacettepe, Ankara 2008.

Balzac H. (1874). Les médecins de campagne. Oeuvres complètes, tome 13.djvu/311. 1874.

Genette G. (1972). Figures: essais. Paris 1972.

Goldenstein J. P. (1980). Pour lire le roman: initiation à une lecture méthodique de la fiction narrative. Bruxelles 1980.

Greimas A. J. \& Courtès J. (1993). Dictionnaire raisonné de la théorie du langage. Paris 1993.

Groupe d'Entrevernes. (1979). Analyse sémiotique des Textes. Lyon 1979.

Günay D. (2013). Metin Bilgisi. İstanbul 2013.

Özel-Coulibaly I. (2012). Etude du Point de vue narratif dans la Signification textuelle. Thèse de master inedite. Dokuz Eylül Üniversitesi, İzmir 2012.

Radiguet R. (1967). Diable au corps. Paris 1967.

\section{Sites internet}

http://faculty.georgetown.edu/spielmag/docs/txt/enonciation.htm, le 3 Février 2018.

http://jv.gilead.org.il/gallica/T0089830.html, le 4 Février 2018.

https://.facebook.com/l.php?u=http\%3A\%2F\%2Fjocelyne.vilmin.free.fr\%2Fwp-

content $\% 2$ FStatut $\% 2520$ du $\% 2520$ narrateur $\% 2520$ et $\% 2520$ point $\% 2520$ de $\% 2520$ vue $\% 2520$ ou $\% 2520$

focalisation\%2520dans\%25201e\%2520roman.pdf\&h=ATNZtOgn1Nn5iIx1DlXbt2XaHIeApSA4bRH

WQYL0dcbug3nSDvh6imNGz8Q8gq7jeG85JNpArylPoqWvkmwmIeUApwRaOvpm-

tesirLZJ4AnrG90yT9D4NJ8xFf9BEuDDaT-0AhZbClGMAcz, le 05 Février 2018.

http://journals.openedition.org/narratologie/6795 Jean-Marc Limoges, «De l'écrit à l'écran», Cahiers de Narratologie [En ligne], 25 | 2013, mis en ligne le 20 décembre 2013, consulté le 08 février 2018. 